\title{
Computer Simulation of Simultaneous Evolution of Individual Texture Components During Recrystallization of an IF Steel
}

\author{
Simone Carreiro de Oliveira ${ }^{\mathrm{a}}$, Weslley Luiz da Silva Assis ${ }^{\mathrm{a}}$, Elena Villa ${ }^{\mathrm{b}}$, Paulo Rangel Rios ${ }^{\mathrm{a} *}$ \\ ${ }^{a}$ Escola de Engenharia Industrial Metalúrgica de Volta Redonda, Universidade Federal \\ Fluminense - UFF, Av. dos Trabalhadores, 420, CEP 27255-125, Volta Redonda, RJ, Brasil \\ ${ }^{\mathrm{b}}$ Department of Mathematics, The University of Milan - UNIMI, Via Saldini 50, 20133, Milano, Italy
}

Received: March 30, 2013; Revised: May 20, 2013

\begin{abstract}
Computer simulation of simultaneous evolution of individual texture components during recrystallization of an IF steel is carried out. A new methodology has been developed that takes as its starting point experimental data of each texture component evolving during recrystallization. In particular, Magnusson et al. data on IF steel was analyzed in a previous work with the help of the exact analytical tools developed by Rios and Villa for simultaneous transformations. From this analysis parameters such as number of nuclei of each texture component per unit of volume could be obtained and were employed as input for the present computer simulation. From this input 3-d microstructures could then be generated. The methodology proposed here combines experimental data, exact analytical methods and computer simulation and may be employed to extract the maximum information from the experimental data.
\end{abstract}

Keywords: microstructure, kinetics, recrystallization, computer simulation, analytical methods, simultaneous transformations

\section{Introduction}

Recrystallization in metals may be regarded as the progression of several simultaneous nucleation and growth transformations ${ }^{1}$. Each transformation corresponds to the evolution of an individual texture component possessing its own nucleation and growth characteristics. As a consequence, one would like to develop a computer model to simulate the of recrystallization in which each texture component nucleation and growth experimental parameters could be inserted into the simulation. This, however, would require that experimental nucleation and growth data for every texture component participating in the recrystallization process were available. Considering that during recrystallization of, for example, an IF steel more than one texture component may evolve simultaneously the task of measuring the nucleation and growth of each individual component looks daunting.

Nonetheless two key developments, one experimental, another theoretical, make this task possible. The first has been around for some time it is the Electron backscatter diffraction (EBSD) technique that permits the measurement of individual orientations of the grains and thus makes it possible to measure the growth velocity of each texture component by conjugating orientation measurements of individual grains and usual stereological measurements (volume fraction and interfacial area per unit of volume) obtainable from planar sections by quantitative metallographic techniques. This has been done by Magnusson et al. ${ }^{2}$ and their dataset will be extensively used in this paper. Unfortunately, there has been no follow up to their excellent work meaning that no

*e-mail: prrios@id.uff.br further measurements of this kind have been carried out, perhaps because the potential of their data as the basis for a 3-d computer simulation of texture evolution could not be perceived at that time.

The second development has taken place only recently ${ }^{3}$ although earlier attempts had been made ${ }^{4,5}$. Such development was an exact mathematical framework derived by Rios and Villa $^{3}$ to treat simultaneous and sequential transformations. Rios and Villa ${ }^{3}$ methodology permits extracting data for the behavior of an individual transformation from measurements carried out in a situation in which such a transformation is taking place together with other transformations, in other words several transformations are taking place simultaneously. Rios and Villa ${ }^{3}$ methodology will be briefly described in the next section.

Putting together Magnusson et al. ${ }^{2}$ experimental data and the exact mathematical technique of Rios and Villa ${ }^{3}$ it is in principle possible to obtain the individual data for each texture component and use this data as input for a 3-d computer simulation of texture evolution during recrystallization of an IF steel. This is the task we endeavor to accomplish in this work. In section 2 we briefly describe Rios and Villa ${ }^{3}$ methodology and in section 3 we summarize our previous analytical work with Magnusson et al. ${ }^{2}$ data that provided the input for the computer simulation. These sections were included with the purpose to give the reader an all-round grasp of the methodology we are proposing here.

Throughout this paper "volume fraction transformed", $V_{V}$, and "volume fraction recrystallized" as well as "transformation" and "recrystallization" are used 
interchangeably. This is done because even though in this paper we focus on recrystallization the methodology provided here is quite general and may be applied when instead of two texture components one has two simultaneous arbitrary phase transformations.

\section{Analytical Modeling of Simultaneous Transformations}

The first point of Rios and Villa ${ }^{3}$ methodology is that it is necessary to make a distinction between "experimental" and "theoretical" quantities.

The experimental volume fraction of a transformation $i$ is denoted by $V_{V i}^{*}(t)$. It is the volume fraction of transformation $i$ that is directly measured from the transformed microstructure. This is exemplified in Figure 1a where only two transformations, 1 and 2, take place simultaneously. Notice that in this case, as shown in Figure 1a, there is impingement between transformations 1 and 2 and (not shown in Figure 1a) between transformations 1 and 1 and between 2 and 2 . Notice that the total experimental volume fraction, $V_{V}$, is equal to the sum of the two experimental volume fractions, $V_{V}=V_{V 1}^{*}+V_{V 2}^{*}$.

The theoretical volume fraction of a transformation $i$ is denoted by $V_{V i}(t)$. The theoretical volume fraction associated with a transformation $i, V_{V i}(t)$, is the value of $V_{V i}(t)$ at a certain time $t$ if the transformation $i$ were the only transformation taking place. This is exemplified in Figure 1b when only two transformations take place simultaneously. In this case, as depicted in Figure 1b, there is no impingement between 1 and 2 transformations, but there is impingement (not shown in Figure 1b) between 1 and 1, and also between 2 and 2 transformations. In Figure $1 \mathrm{~b} V_{V 1}(t)$ is the theoretical volume fraction of transformation 1 because it is a measure (in an engineering sense) of to what extent transformation 1 would take place as a function of time if no transformation 2 would take place. An analogous idea applies to the concept of theoretical volume fraction of transformation 2 .

Clearly, a key objective of a methodology to study simultaneous transformations is to "extract" theoretical quantities of individual reactions from experimental quantities. Once this is achieved one may study each transformation in isolation using all analytical and computational models developed for individual reactions. Rios and Villa ${ }^{3}$ developed an exact analytical mathematical methodology to perform this task. Their main results can be summarized in the three equations presented below.

$V_{V}(t)=1-\Pi_{i=1}^{i=n}\left(1-V_{V i}(t)\right)$

Where $V_{V}$ is the total volume fraction, so Equation 1, is a way to obtain the total experimental volume fraction from the theoretical volume fractions of the individual transformations.

$V_{V i}(t)=1-\exp \left(-\int_{0}^{t} \frac{1}{1-V_{V}(s)} \frac{\partial V_{V i}^{*}}{\partial s} d s\right)$

Equation 2 is the key equation of the methodology. It allows obtaining the theoretical volume fraction of a certain transformation $i$ solely in terms of experimentally measured quantities.
$V_{V i}^{*}(t)=1-\exp \left(-\int_{0}^{t} \frac{1-V_{V}(s)}{1-V_{V i}(s)} \frac{\partial V_{V i}}{\partial s} d s\right)$

Equation 3 allows obtaining the experimental volume fraction of a certain transformation $i$ as a function of the theoretical volume fractions of the individual transformations. Equations 1-3 remain valid provided that the nucleation and growth of the simultaneous transformations are independent of one another. For details, the reader is referred to the original paper ${ }^{3}$.

\section{Analytical Treatment of the Experimental Results of Magnusson et al. ${ }^{2}$}

Magnusson et al. ${ }^{2}$ studied the growth rates of the different texture components of an IF steel. They measured the growth rate of the " $\gamma$-fiber" or " $\gamma$ " components and the

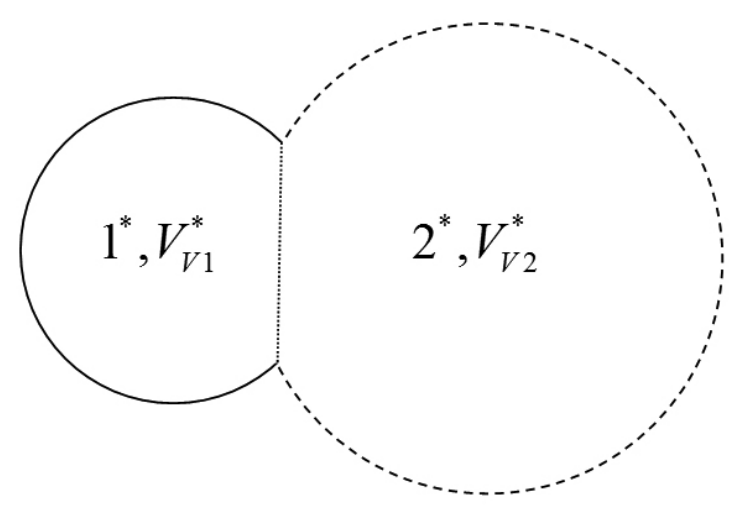

$1 a$

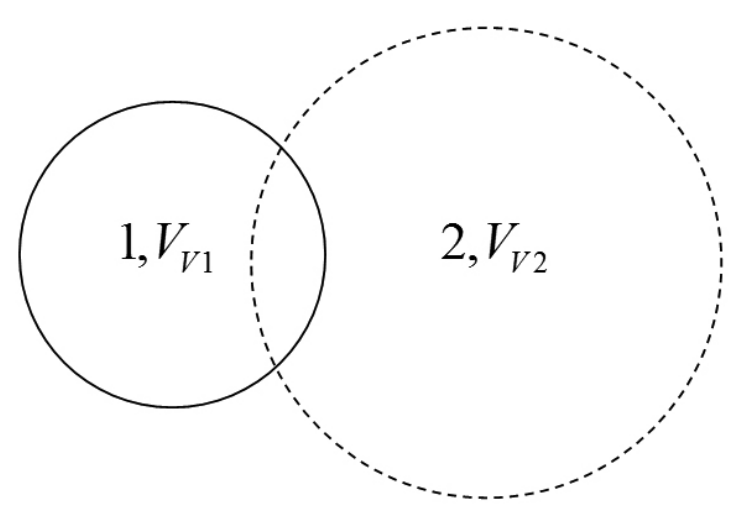

$1 b$

Figure 1. Illustration of the definitions of experimental (1a) and theoretical (1b) quantities for two transformations; (1a) The "visible" or "experimental" transformed region of each reaction is indicated by $1 *$ and $2 *$. The volume fraction of $1 *$ and $2 *$ are $V_{V 1}^{*}$ and $V_{V 2}^{*}$, respectively. $V_{V 1}^{*}$ and $V_{V 2}^{*}$, are called "experimental volume fractions". The dotted line on the right hand side figure is the boundary between $1 *$ and $2 * ;(1 b)$ The "theoretical" transformed region of each reaction is labeled 1 and 2 . The volume fractions of 1 and 2 are $V_{V 1}$ and $V_{V 2}$, respectively. $V_{V 1}$ and $V_{V 2}$ are called "theoretical volume fractions". 
growth rate of what they called "other" or " $o$ " components. Magnusson et al. ${ }^{2}$ do not report the experimental errors but judging from the techniques they used, it is reasonable to expect their relative experimental errors to be at roughly $\pm 10 \%$, perhaps even higher at the beginning of the recrystallization. Therefore, for the sake of comparison with analytical models and computer simulation, error bars of this magnitude were inserted in the graphs containing their data. For details of their IF steel, experimental techniques and results the reader is referred to their work. Rios et al. ${ }^{6}$ applied Rios and Villa ${ }^{3}$ methodology, briefly reviewed in the previous section, to the results of Magnusson et al. ${ }^{2}$. The analysis was carried out by Rios et al. ${ }^{6}$ as follows. First, the experimental data of Magnusson et al. ${ }^{2}$ were best-fitted with conveniently chosen functions. This was done so that the integrations and derivatives required by Equation 2 could be carried out more easily. Second, Equation 2 was used to obtain the theoretical volume fraction of the $\gamma$-fiber components as a function of time as well as the theoretical volume fraction of other components as a function of time, from the experimental quantities reported by Magnusson et al. ${ }^{2}$. Third, Rios et al..$^{6}$ analyzed the theoretical volume fraction of the $\gamma$-fiber and of the other components obtained by Equation 2 with the help of usual analytical models for recrystallization, for example, supposing site-saturated nucleation ${ }^{7,8}$. Rios et al. ${ }^{6}$ employed the simplest assumptions regarding nucleation, namely, that the nucleation of both $\gamma$ and $o$ components was site-saturated. The growth velocity of $\gamma$-fiber and other components was taken to be equal to the velocity experimentally measured by Magnusson et al. ${ }^{2}: G_{\gamma}=0.14 t^{-0.58} \mu \mathrm{m} / \mathrm{s}$ and $G_{o}=0.08 t^{-0.49}$ $\mu \mathrm{m} / \mathrm{s}$. For site-saturation, with uniform randomly located nuclei ${ }^{9}{ }^{10}$ one can write

$V_{V \gamma}^{m}=1-\exp \left(-\frac{4 \pi N_{V \gamma}^{m}}{3}\left(\int_{s=0}^{s=t} G_{\gamma}(s) d s\right)^{3}\right)=1-\exp \left(-3.92 \times 10^{-5} t^{1.26}\right)$

$V_{V o}^{m}=1-\exp \left(-\frac{4 \pi N_{V o}^{m}}{3}\left(\int_{s=0}^{s=t} G_{o}(s) d s\right)^{3}\right)=1-\exp \left(-2.57 \times 10^{-6} t^{1.53}\right)(5)$

Where $V_{V \gamma}^{m}(t)$ and $V_{V o}^{m}(t)$ are the volume fractions of the $\gamma$-fiber and other components, respectively, obtained by best-fitting the site-saturated nucleation and growth model to the theoretical volume fraction. The fitting parameters were the number of nuclei per unit of volume of the $\gamma, N_{V \gamma}^{m}$, and $o, N_{V o}^{m}$, components. The best fitting gives $N_{V \gamma}^{m}=0.000253$ nuclei $/ \mu \mathrm{m}^{3}$, with a correlation coefficient equal to $R^{2}=0.997$ and $=0.000159$ nuclei $/ \mu \mathrm{m}^{3}$ with a correlation coefficient equal to $R^{2}=0.979$.

The total volume fraction obtained by the model, $V_{V}^{m}$, may be obtained by the superposition principle, Equation 3, in the same way as the theoretical quantities ${ }^{6}$

$$
\begin{aligned}
& V_{V}^{m}(t)=1-\left(1-V_{V \gamma}^{m}(t)\right)\left(1-V_{V o}^{m}(t)\right)= \\
& 1-\exp \left(-\left(3.92 \times 10^{-5} t^{1.26}+2.57 \times 10^{-6} t^{1.53}\right)\right)
\end{aligned}
$$

These three curves are depicted together with the theoretical quantities in Figures 2, 3 and 4. The agreement between model and theoretical volume fractions is good for

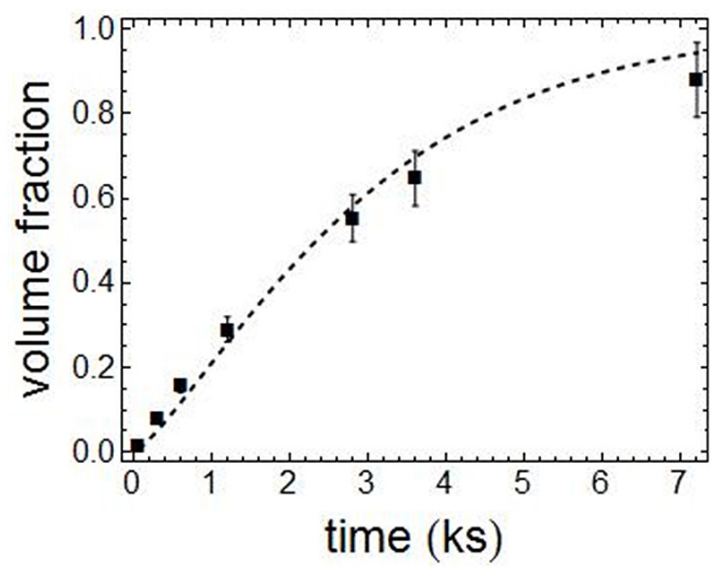

Figure 2. Comparison between model (dashed line), Equation 4, and theoretical volume fraction (filled squares) obtained from experimental data of $\gamma$-fiber orientations by Equation 2 .

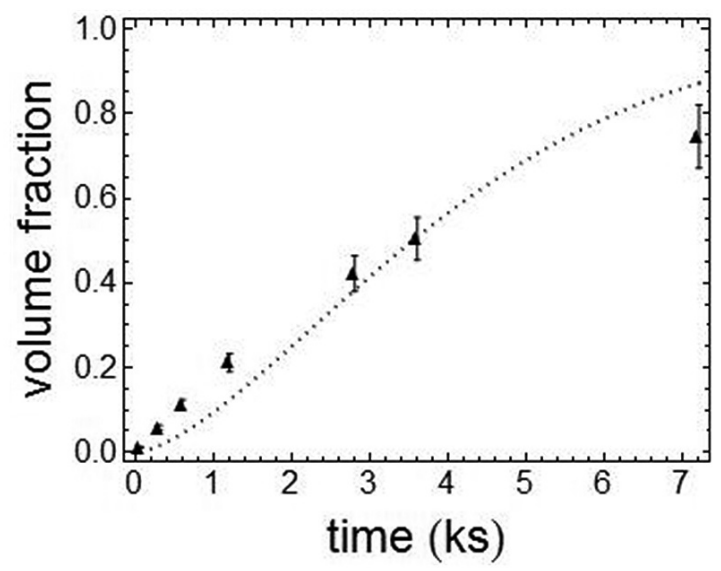

Figure 3. Comparison between model (dotted line), Equation 5, and theoretical volume fraction (filled triangles) obtained from experimental data of other orientations by Equation 2 .

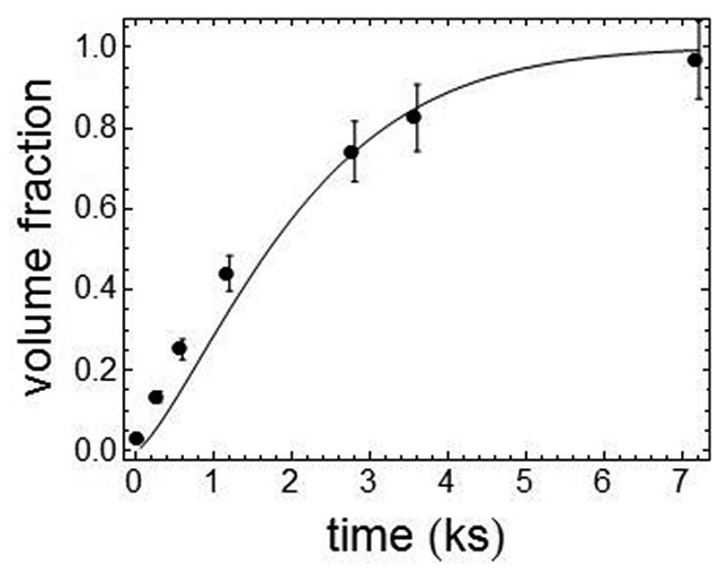

Figure 4. Comparison between model (solid line), Equation 6, and the total theoretical volume fraction (filled circles). 
transformation 1 and reasonable for transformation 2 . This point will be taken up again later in this paper.

Rios et al. ${ }^{6}$ result suggests that the site-saturated model provides a reasonable description of the behavior of the kinetics of the $\gamma$-fiber and of other texture components in the IF steel studied by Magnusson et al. ${ }^{2}$. The analysis provides quantitative estimates for the number of nuclei per unit of volume.

Magnusson et al. ${ }^{2}$ experimental data plus Rios et al. ${ }^{6}$ analytical approach give a good quantitative description of the main parameters of the IF steel studied by Magnusson et al. ${ }^{2}$. Combining both studies one has enough information about nucleation and growth to carry out a computer simulation of the IF steel and thus obtain the full 3-d microstructure evolution during the recrystallization of the IF steel with the simultaneous and competitive evolution of the $\gamma$-fiber and of other texture component. This is what will be done in the sequel.

\section{Computer Simulation Methodology}

The simulation methodology used here was employed before $^{11,12}$. Figure 5 shows the principle underlying the methodology. On the left hand side Figure 5 shows a point $\mathrm{x}$ inside a ball of radius $R=G t$ centered in x (solid line). This ball is called the causal cone ${ }^{10}$ of $\mathrm{x}$. No nuclei are inside the ball. As a consequence, no transformed region can overtake $\mathrm{x}$ that remains untransformed. In contrast, the right hand side of Figure 5 shows the situation in which there is one nucleus inside the ball centered in x with radius $G t$. At time $t$ the transformed region originated at the nucleus has grown to a ball of radius $R=G t$ centered on the nucleus (dashed line) encompassing the point $\mathrm{x}$. In other words $\mathrm{x}$ is now inside the transformed region. Notice that at least one nucleus must be present within the ball centered in $\mathrm{x}$ so that $\mathrm{x}$ is transformed, but more than one nucleus may be located within this ball.

In order to implement this idea a FORTRAN program was written. The program was parallelized with OPEN

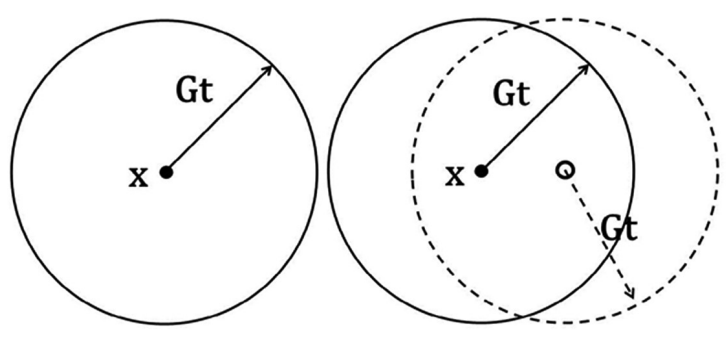

Figure 5. On the left hand side of the figure point $\mathrm{x}$ is inside a ball of radius $R=G t$ centered in $\mathrm{x}$ (solid line). This ball is called the causal cone of $x$. No nuclei are inside the ball. As a consequence, no transformed region can overtake $\mathrm{x}$ that remains untransformed. In contrast, the right hand side of the figure shows a ball centered in $\mathrm{x}$ with one nucleus inside it. At time $t$ the transformed region originated at the nucleus has grown to a ball of radius $R=G t$ (dashed line) encompassing the point $\mathrm{x}$ so that it is inside the transformed region. Notice that at least one nucleus must be present within the ball centered in $\mathrm{x}$ so that $\mathrm{x}$ is transformed, but more than one nucleus may be located within this ball.
MP and could be run in a matter of hours in a dual Xeon machine with sixteen cores per processor. A matrix of dimensions $300 \times 300 \times 300$ was used to represent the "specimen" in which the transformation was taking place. The usual periodic boundary conditions were used in all cases. Two phases, g and other, were simulated to grow simultaneously. The growing velocity and number of nuclei per unit of volume of each phase in the simulation were taken to be equal to the experimental velocity measured by Magnusson et $\mathrm{al}^{2}$ and the number of nuclei per unit of volume of each individual texture component extracted from the analysis carried out on the experimental data of Magnusson et al. ${ }^{2}$ described above. This will become clearer later in this paper. Units were assigned so that the $300 \times 300 \times 300$ matrix corresponded to cubic specimen of size $1 \times 1 \times 1 \mathrm{~mm}$.

\section{Computer Simulation and Experimental Data}

Figures 6-8 show the transformed volume fraction as function of time obtained by computer simulation compared with the experimental result from Magnusson et al. ${ }^{2}$. Figure 6 exhibits the total volume fraction transformed as a function of time adding up the volume fraction transformed of the $\gamma$-fiber plus the volume fraction transformed of the other texture component. Figure 7 depicts the "experimental" volume fraction transformed of the $\gamma$-fiber components and Figure 8 depicts the volume fraction transformed of the other components. Both Figure 7 and 8 exhibit plots of experimental values of computer simulation and of the experimental results of Magnusson et al. ${ }^{2}$. Notice that the terminology "experimental" used in connection with computer simulation might cause some confusion but here it is used in the specific sense defined by Rios and Villa ${ }^{3}$ in their model, see section 2 .

The agreement between computer simulation and experimental result is reasonable. One possible source of discrepancy between simulation and Magnusson et al. ${ }^{2}$ experimental data could be that the site-saturation model

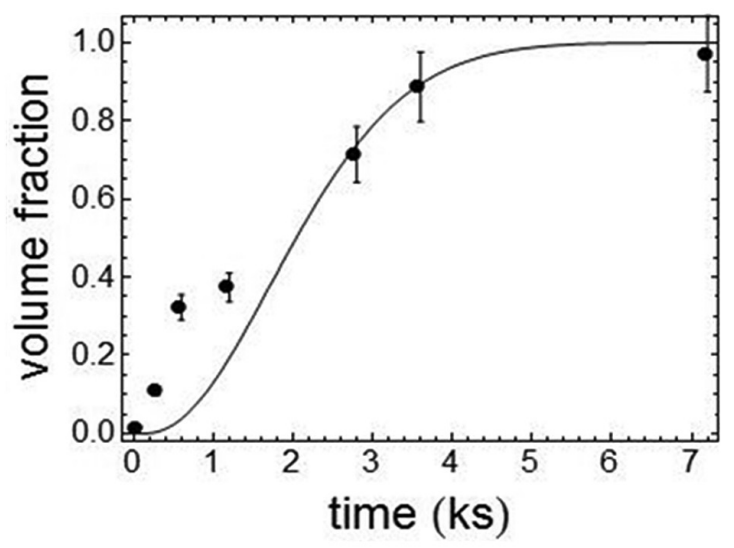

Figure 6. Comparison between total volume fraction transformed obtained by computer simulation (solid line) and experimental data from Magnusson et al. ${ }^{2}$ (filled markers). 
with nuclei uniform randomly located in space evolving with a single velocity that was used as the starting point of the simulation is an idealized view of the real situation. Magnusson et al. ${ }^{2}$ lumped under the name of $\gamma$-fiber and of other orientations what could be in fact two or more simultaneous transformations in each case. Therefore may be a single velocity and a single number of nuclei per unit of volume, implying a single volume fraction against time curve might not be an accurate description of the behavior of the texture evolution. Indeed, a single experimental velocity representing the $\gamma$-fiber and the other orientations, as reported by Magnusson et al. ${ }^{2}$, is probably an approximation. In the analytical work, particularly in the case of the other orientations the agreement of the site-saturation model was noticeably worse for the other orientation than for the $\gamma$-fiber orientation, see Figure 3. It is worthy of note that In the analytical modeling only mean values are employed but in the simulation the nuclei are randomly spread in space. This spread in the nuclei location introduces a variance

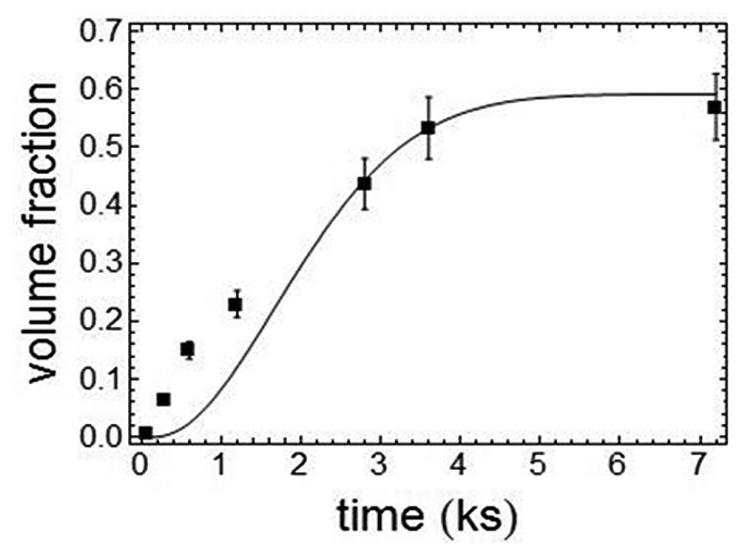

Figure 7. Comparison between volume fraction transformed of $\gamma$-fiber orientations obtained by computer simulation (solid line) and experimental data from Magnusson et al. ${ }^{2}$ (filled markers).

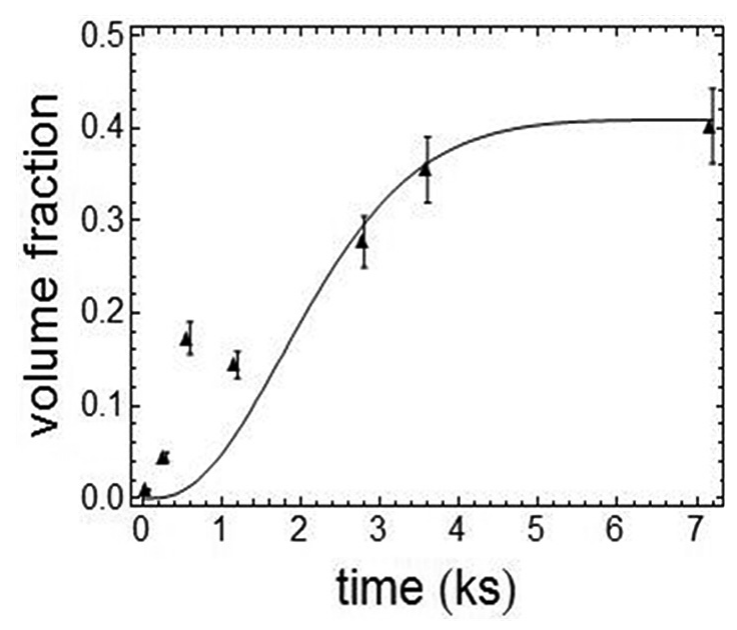

Figure 8. Comparison between volume fraction transformed of other orientations obtained by computer simulation (solid line) and experimental data from Magnusson et al. ${ }^{2}$ (filled markers). in space and makes it harder to assess what is the overall effect of an initial error on the mean values. This is probably even more difficult for a simulation involving two phases because a two phase simulation is much more complex than a simulation involving a single phase. Another factor that could be observed is that the experimental volume fraction against time curves of Magnusson et al. ${ }^{2}$, not shown in this paper, have a distinctive exponential not sigmoidal character. This is unusual for recrystallization of steels. May be this characteristic of their curves could be due to experimental errors that prevented a better description of their curves at the beginning of the recrystallization. To speculate about the reason for this is pointless in view of the lack of data. The point is that the computer simulation owing to its construction: uniform randomly located nuclei will produce sigmoidal curves. Therefore, some discrepancy is unavoidable.

Still the agreement between simulation and experiment is acceptable. The simulation will always be limited by the quality and by level of the detail of the experimental data on which it is based and there is not much that can be done but to accept this limitation. Paradoxically, in spite of hundreds or perhaps thousands of papers on recrystallization of IF steels systematic datasets such as that of Magnusson et al. ${ }^{2}$ are hard to come by. Hopefully the availability of a welldefined methodological analytical tool presented here may encourage the production of such data in the future.

\section{Applications of Computer Simulation and Summary of Proposed Modeling Methodology}

A significant advantage of a computer simulation "model" over an analytical model is that the computer simulation is able to generate the microstructures. This is shown in Figures 9 and 10. Figure 9 exhibits a 3 -d view of the computer simulated of the recrystallization of an IF steel taking the experimental nucleation and growth of the individual $\gamma$-fiber and of other texture components as its starting point. From the 3 -d view any microstructural view may be extracted. For example, Figure 10 shows a sequence of more familiar 2-d evolution "micrographs". The 2-d computer simulation micrographs allow us to have an idea of what to expect when looking at real micrographs (of course obtained using EBSD technique) of an IF steel with similar texture. A point that one would not be able to figure out without the computer simulation can be better appreciated in observing the fully transformed micrograph, the last of the sequence, in Figure 10. The higher volume fraction $\gamma$-fiber components appear to surround the lower volume fraction other components. Therefore in the final micrograph one might have a certain impression that the other components are "clustered" and surrounded by the $\gamma$-fiber components. This may lead one to reach erroneous conclusions about the nucleation sites of the $\gamma$-fiber and of other components. In fact in the computer simulation the nuclei of both $\gamma$-fiber and of other components were located uniform randomly in space, there was no nucleation clustering of the other components. 


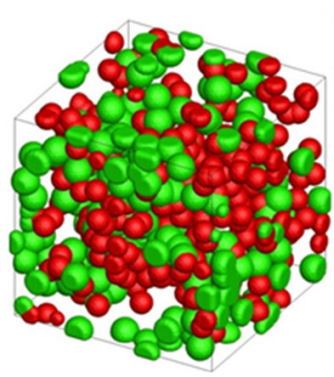

(a)

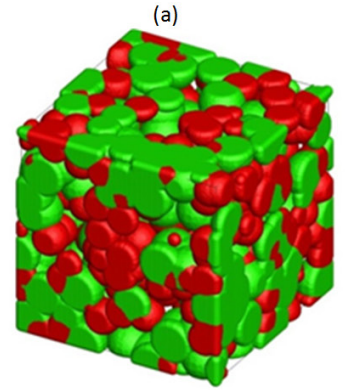

(c)

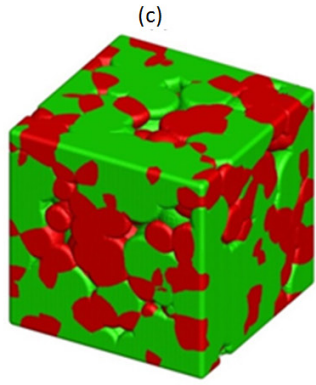

(e)

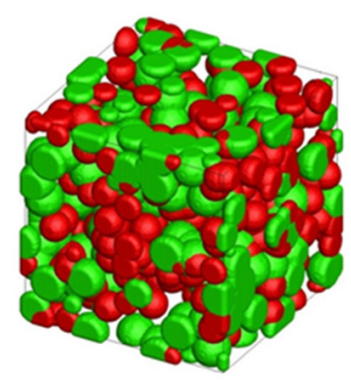

(b)

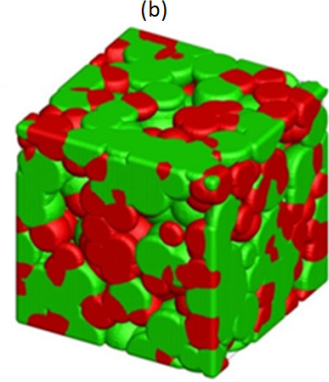

(d)

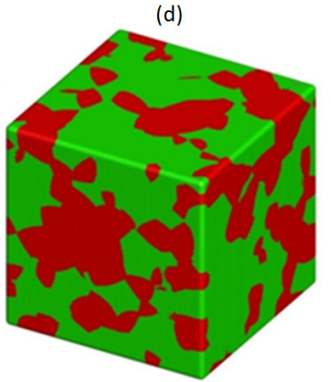

(f)

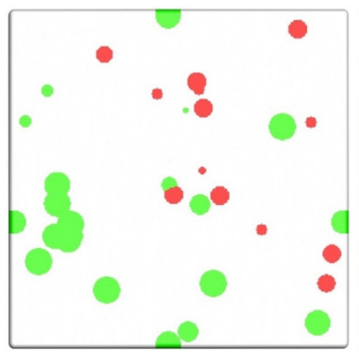

(a)

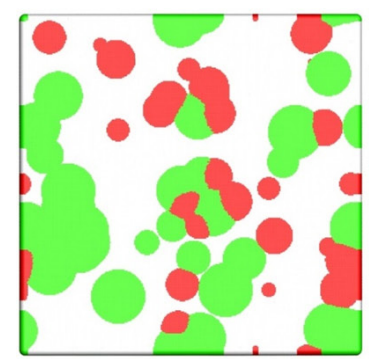

(c)

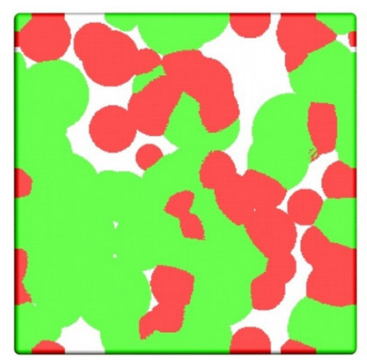

(e)

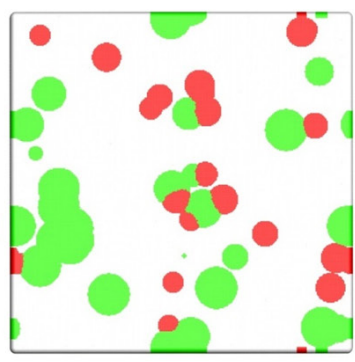

(b)

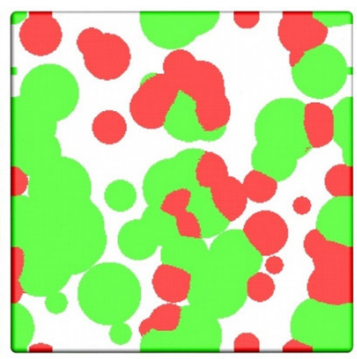

(d)

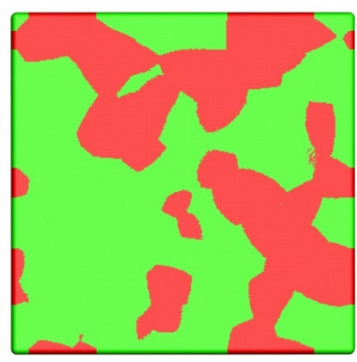

(f)

Figure 9. 3-d of the recrystallization: volume fraction of $\gamma$-fiber ("green") and other ("red") orientations are, respectively,: a)0.03, 0.02 b) $0.12,0.08$ c) $0.25,0.15$, d) $0.36,0.24$, e) $0.48,0.32$ and f) $0.58,0.42$.

In section 5 we discussed in detail the limitations of the computer simulation as a function of the experimental data on which it is based. This is ultimately responsible for the discrepancies found in the earlier part of the kinetics curve shown in Figure 6. This discrepancy finds a parallel in the simulated microstructure when compared with experimentally observed microstructures normally observed in these steels. Experimental microstructures normally show the so-called $\gamma$-bands whereas the simulated microstructures do not. Therefore simulated microstructures are more uniform than the experimental microstructures. Unfortunately, Magnusson et al. ${ }^{2}$ did not report any microstructural information not even a verbal description of the microstructure. Therefore, likewise in section 5 , there is not much one can do to improve the simulation in this regard.

Finally, once one has the computer simulation programmed it is possible to simulate any "what if" scenario that might be helpful to understand how, for example, will the microstructure look like should one manage, by means of

changing the processing, to increase or decrease the number of nuclei per unit of volume of the $\gamma$-fiber/other components. This possibility is depicted in Figure 11. It is worthy of note that one cannot really be sure that the growth velocity of the $\gamma$-fiber/other components will remain unchanged when their proportion changes but Figure 11 at least gives a first approximation. In Figures $6-1061.4 \%$ of the total number of nuclei per unit of volume were nuclei of the $\gamma$-fiber components. In the simulation to generate the fully transformed microstructures of Figure 11 this percentage was changed from $20 \%$ of $\gamma$-fiber nuclei in Figure 11a up to $90 \%$ in Figure 11e. The caption of Figure 11 also contains the final volume fraction of $\gamma$-fiber and other components for each percentage of $\gamma$-fiber nuclei employed in the simulation. In Figure 11 the $\gamma$-fiber components increase its volume fraction from Figure 11a to 11e. Only a visual inspection of Figure 11 is performed here, a full quantitative microstructural analysis would be beyond the scope of this paper. In Figure 11a $\gamma$-fiber components are entirely 


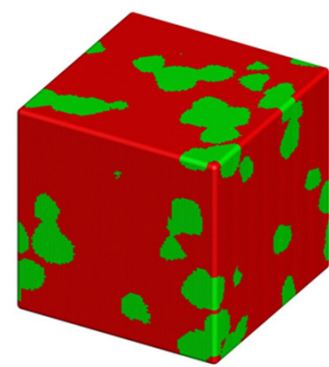

(a)

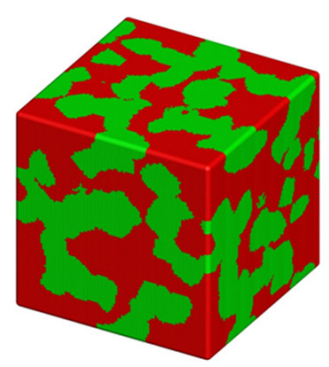

(c)

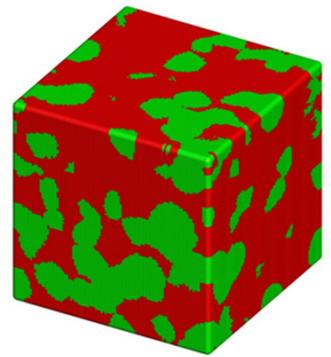

(b)

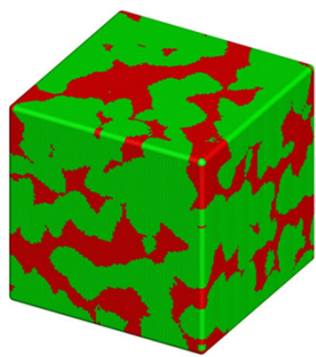

(d)

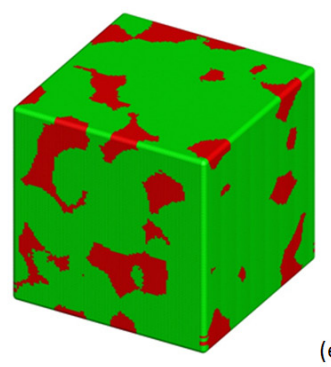

(e)

Figure 11. 3-d fully recrystallized microstructures as a function of the percentage of the total number of nuclei per unit of volume that are nuclei of the $\gamma$-fiber components. The numbers represent the percentage of $\gamma$-fiber orientations nuclei, the volume fraction of $\gamma$-fiber ("green") and other ("red") orientations, respectively: a) $20 \%, 0.20,0.80$ b) $40 \%, 0.36,0.64$ c) $60 \%, 0.56,0.44$, d) $70 \%$, $0.66,0.34$, e) $90 \%, 0.86,0.14$.

surrounded by other components whereas in Figure 11e just the opposite takes place. It is interesting to see where this transition takes place. Visual inspection suggests that a transition takes place between Figures $11 \mathrm{c}(60 \% \gamma$-fiber nuclei) and $11 \mathrm{~d}(70 \% \gamma$-fiber nuclei). In Figure 11c the $\gamma$-fiber components still appear to be surrounded by the other components whereas in Figure 11d it is the other components that appear to be surrounded by the $\gamma$-fiber components. The presence of $\gamma$-fiber components and the strength of the $\gamma$-fiber texture components are normally desirable for better deep drawing properties of the steel sheet. The computer simulation is showing that significant microstructural changes are taking place in the underlying microstructure associated with the changes in the strength of the $\gamma$-fiber texture.

\section{Summary and Conclusions}

A new methodology combining experimental data, exact analytical methods and 3-d computer simulation was put forward to analyze texture evolution during recrystallization from the evolution of the individual texture components. Either step in the proposed methodology is easily reproducible and is described in sufficient detail in this paper and in the references supplied

The main steps of the proposed methodology are:

- To obtain a good experimental dataset from which one may have volume transformed against time curves for each time for each texture component or $r$ as in the present analysis for a "fiber" and its corresponding growth velocity;

- From the experimental data by means of the exact analytical tools (see section 2) one may extract the kinetics of each individual texture component as exemplified in section 3. One may then analyze the individual kinetics with analytical models such as sitesaturation model or constant nucleation model. Notice that the growth velocity does not need to be obtained by fitting as they were obtained experimentally;

- Once the nucleation model and the number of nuclei per unit of volume have been found rom experimental data one can use these as the starting point for the computer simulation. The computer simulation results may then be checked against the original experimental data;

- The computer simulation may be used to produce computer generated 3-d and 2-d microstructures and/or time dependent. Furthermore, the computer simulation may employed to investigate a diversity of "what if" scenarios, for example, by changing the number of nuclei per unit of volume and the perhaps even the velocity.

Finally, it is worth of note that the ever growing complexity of modern materials science demands that, whenever possible, all tools be combined to face the problems: experiments, analytical tools, computer simulation. This is not always easy and not always possible. We believe that in this paper we demonstrated that this is possible but each part must be aware of the needs of the other, i.e. the simulation needs systematic data whereas the experimentalist needs a "experimentally friendly" theoretical methodology in order to make the most of the potential of the combined techniques.

\section{Acknowledgments}

P. R. Rios, S. C. Oliveira and W L. S. Assis are grateful to Conselho Nacional de Desenvolvimento Científico e Tecnológico, CNPq, and to Fundação de Amparo à Pesquisa do Estado do Rio de Janeiro, FAPERJ, for financial support. 


\section{References}

1. Lauridsen EM, Poulsen HF, Nielsen SF and Jensen DJ. Recrystallization kinetics of individual bulk grains in $90 \%$ coldrolled aluminum. Acta Materialia. 2003; 51(15):4423-4435. http://dx.doi.org/10.1016/S1359-6454(03)00278-7

2. Magnusson H, Jensen DJ and Hutchinson B. Growth rates for different texture components during recrystallization of IF steel. Scripta Materialia. 2001; 44(3):435-441. http://dx.doi. org/10.1016/S1359-6462(00)00621-7

3. Rios PR and Villa E. Simultaneous and sequential transformations. Acta Materialia. 2011; 59(4):1632-1643. http://dx.doi.org/10.1016/j.actamat.2010.11.030

4. Vandermeer RA and Jensen, DJ. Modeling microstructural evolution of multiple texture components during recrystallization. Acta Metallurgica et Materialia. 1994; 42(7):2427-2436. http:// dx.doi.org/10.1016/0956-7151(94)90321-2

5. Jones SJ and Bhadeshia HKDH. Kinetics of the simultaneous decomposition of austenite into several transformation products. Acta Materialia. 1997; 45(7):2911-2920. http:// dx.doi.org/10.1016/S1359-6454(96)00392-8

6. Rios PR, Villa E and Oliveira S C. New methodology to model simultaneous and sequential reactions: main results and applications. Materials Science Forum. 2012; 706-709:149-156. http://dx.doi.org/10.4028/www.scientific.net/MSF.706-709.149
7. Vandermeer RA, Masumura RA and Rath BB. Microstructural paths of shape-preserved nucleation and growth transformations. Acta Metallurgica et Materialia. 1991; 39(3):383-389. http:// dx.doi.org/10.1016/0956-7151(91)90317-T

8. Rios PR and Villa E. Transformation kinetics for inhomogeneous nucleation. Acta Materialia. 2009; 57(11):1199-1208. http:// dx.doi.org/10.1016/j.actamat.2008.11.003

9. Jensen DJ. Simulation of recrystallization microstructures and textures: Effects of preferential growth. Metallurgical Materials Transactions. 1997; 28A(1):15-25. http://dx.doi. org/10.1007/s11661-997-0079-5

10. Rios PR, Villa E, Assis WLS and Ribeiro TCS. Kinetics of transformations nucleated on random parallel planes: analytical modelling and computer simulation. Modelling Simulation Materials Science Engineering. 2012; 20:035017. http://dx.doi. org/10.1088/0965-0393/20/3/035017

11. Vandermeer RA and Rath BB. Modeling recrystallization kinetics in a deformed iron single crystal. Metallurgical Transactions A. 1989; 20A(3):391-401.

12. Salazar TC, Assis WLS and Rios PR. Simulation of Recrystallization in Iron Single Crystals. Materials Research. 2008; 11(1):109-115. http://dx.doi.org/10.1590/ S1516-14392008000100021 